\title{
Numerical simulation of positive-potental conductors in the presence of a plasma and a secondary-emitting dielectric
}

\author{
R. L. Kessel, R. A. Miurray, R. Hetzel, and T. P. Armstrong \\ University of Kansas, Lawrence, Kansas 66045
}

(Received 26 September 1984; accepted for publication 7 January 1985)

A $2 \frac{1}{2}$-dimensional, cylindrically symmetric particle in cell program is used to simulate the interaction of a plasma with a positively biased disk covered by a dielectric material (Kapton). Several runs using different combinations of bias voltages and secondary electron yields are discussed. Special attention is paid to the role of the secondaries as the dielectric surface changes from a negative potential to become increasingly positive. This is termed the "snapover" process. The contribution due to secondaries is examined by plotting the distribution (number versus position) of the particles emitted by the impacting on the dielectric. These plots show that secondaries are drawn back into the dielectric as well as the conductor in the case of snapover. Mechanisms and experimental implications for these particular distributions are discussed.

\section{NTRODUCTION}

The importance of secondary emission to current collected by a conducting disk partially covered by a dielectric material has been discussed by Stevens. ${ }^{1}$ He suggested that plasma electrons were accelerated into the dielectric material (Kapton) and generated secondaries which enhanced the current drawn to exposed regions of the conductor (pinholes). It was supposed that the secondaries were not an important factor until the voltage reached a certain value. "At low, positive applied potentials the Kapton surface assumed a slightly negative voltage. As the applied voltage exceeded 100 volts, the surface voltage on the Kapton changed: It became more and more positive until the whole surface was strongly positive." This positive potential implied that more secondaries were being emitted, and this effect was strong enough to enhance the current.

The importance of secondary emission has been explored by Brandon ${ }^{2}$ and Brandon et al. ${ }^{3}$ in a computer simulation of the experiment by Stevens. 'The plasma simulation code is a further development of the $2 \frac{1}{2}$-dimensional program described previously. ${ }^{4-7}$ Electrons striking the dielectric may emit secondaries. The ratio of secondary electron current to primary electron current is determined by fits to ex-

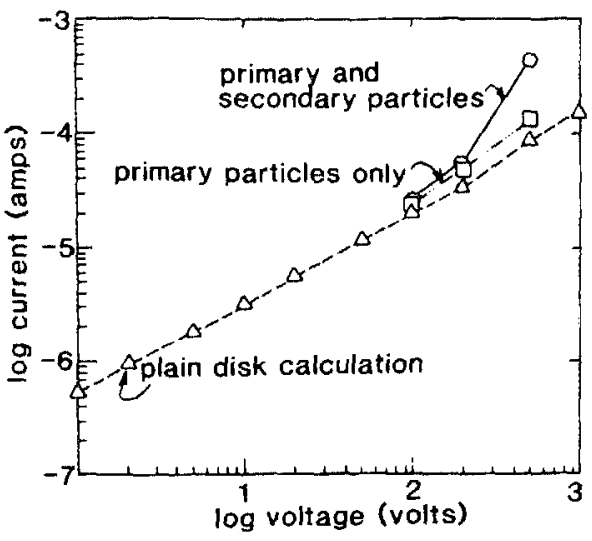

FIG. 1. Current enhancement for the disk and dielectric configuration due to the collection of secondary-emitted particles. perimental data presented by Haffner, ${ }^{8}$ and is given by the expression

$$
\frac{J_{e}^{s}(V)}{J_{e}(V)}=K\left(e^{-a V}-e^{-b v}\right) .
$$

For Kapton the values of $K, a$, and $b$ are $3.5,2.0 / \mathrm{keV}$, and $15.0 / \mathrm{keV}$, respectively.

Bradon et al. described the "pinhole" effect as "the collection of electron current by conductors surrounded by dielectric material in excess of the current which would be collected by a similar sized conductor not surrounded by a dielectric." ${ }^{3}$ They suggested two possible mechanisms to explain the increase in current: electron trajectory focusing as the potential of the conductor spreads into the dielectric surface, and charge hopping along the dielectric surface toward the conductor due to secondary emission. The current enhancement due to the two mechanisms is shown in Fig. 1 (after Brandon et al. ${ }^{3}$ ). An increase in the current is seen when a dielectric is added to the plain conductor, and a further increase is evidenced when secondary emission is included in the calculation. These results show that for voltages just over the first crossover point, the effects of charge focusing and secondary electron emission are about equal. For higher voltages, the increase in current due to collection of secondary particles is the dominant mechanism.

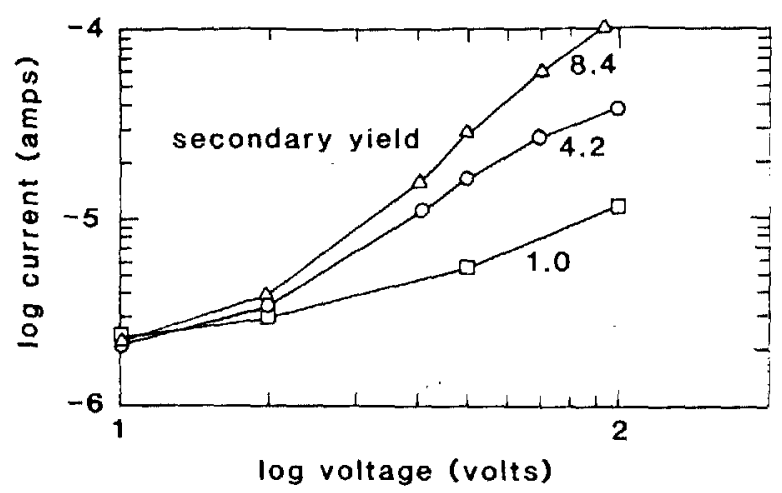

FIG. 2. Current-voltage characteristic curves for positive voltages with variations in the maximum of emitted secondary electrons for an incident primary electron (secondary yield). 


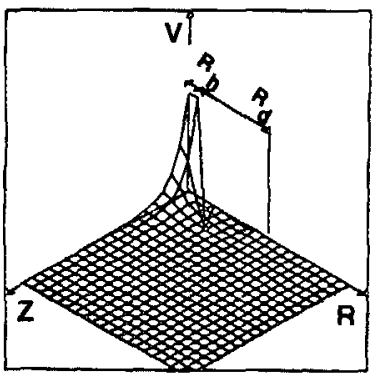

case 1

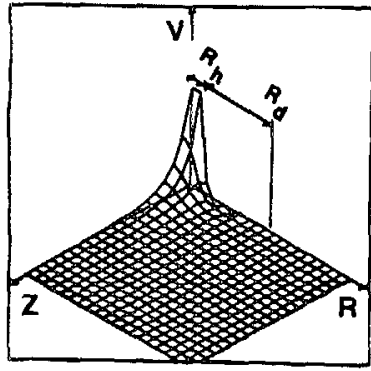

case 3

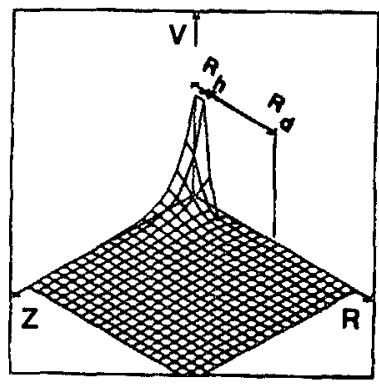

case 2

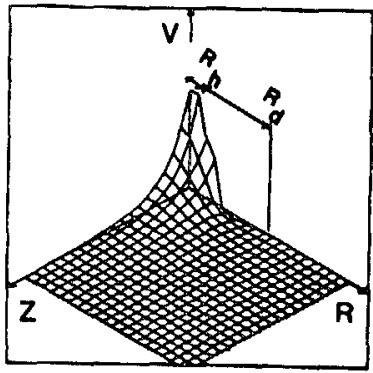

case 4
FIG. 3. Potential snapshots of the four cases at equilibrium. The upper left has a voltage of $40 \mathrm{~V}$ and the s yield of 4.2 . The upper right has a voltage of $70 \mathrm{~V}$ and a s yield of 4.2 . The lower left has a voltage of $40 \mathrm{~V}$ and a s yield of 8.4. The lower right has a voltage of $70 \mathrm{~V}$ and a s yield of 8.4.

Secondary yield is defined as the maximum number of electrons scattered from the dielectric surface per incoming primary electron. Figure 2 shows current-voltage curves with different values of secondary yield. The increase of the current with increasing secondary yield is obvious. A more important effect is that the voltage of the onset for the increase in collected current, with reference to the plain disk, decreases with increasing secondary yield. This onset corresponds to the place in which the voltage on the dielectric surface first becomes positive (Fig. 3, upper right) and has been termed "snapover." 3 The yield of the secondaries has an effect on the voltage at which snapover occurs.

\section{NUMERICAL MODEL}

The objective of this study is to further examine the importance of secondaries by using enhanced diagnostics.
The simulation code retains information about where primaries impact, where secondaries are generated, and where these secondaries impact. Parametric studies include varying the voltage and secondary yield, and examining these impacts and emissions. Four cases have been examined in detail, a combination of two values of peak secondary yield (s yield) and two values of voltage. In the first case the values of 4.2 and 40 were used for the s yield and voltage, respectively; snapover did not occur. This case was included for comparative purposes. The next two cases are both at the onset of snapover. The s yields and voltages were 4.2 at $70 \mathrm{~V}$, and 8.4 at $40 \mathrm{~V}$. Also included was the case well past snapover, $\mathrm{s}$ yield of 8.4 at $70 \mathrm{~V}$.

The geometry and boundary conditions are described as follows. The cylindrical simulation region is divided into a numerical grid which is used to calculate the potentials and fields of the problem. Each cell represents a ring of space due to the rotational symmetry about the $Z$ axis. The outer boundaries, large $R$ and large $Z$, are assumed to be removed far enough from the conduction disk that the potential can be set to zero. Thus, the plasma is Maxwellian at the outer boundaries, and particles are added to the simulation according to the value of the random thermal current. Particles are lost from the simulation whenever their orbits cross one of these boundaries. In all four cases discussed here a $20 \times 20$ calculational grid is used. Along the $R$ axis lie the conductor and dielectric covering (assumed thin) and the ground plate extending from the edge of the conductor to the end of the simulation space. The first two cells are uncovered conductor, the next eight cells are conductor covered by dielectric, and the last ten cells represent the ground plate. Depending on where particles cross this boundary, they are either added to the current, added to the charge on the dielectric surface (in which case they can emit secondaries), or lost from the simulation.

The results presented are the equilibrium configurations. A simulation is considered to be in equilibrium if several conditions are met. The charge on the dielectric surface must have settled down to a constant value (small fluctuations around this value allowed). This settling down must also be true for the number of particles (both species) and their kinetic energies. The last criterion is a steady potential over the entire simulation space. The four cases each took a different number of time steps to reach equilibrium, less for the cases of lower voltage or lower secondary yield. How-

TABLE I. Various percentages due to impacts and emissions along the conductor and first few dielectric grid cells.

\begin{tabular}{|c|c|c|c|c|c|c|}
\hline & \multirow[b]{2}{*}{$\begin{array}{l}\text { Percent of } \\
\text { Current due } \\
\text { to secondaries }\end{array}$} & \multicolumn{2}{|c|}{ Conductor } & \multicolumn{3}{|c|}{ Dielectric } \\
\hline & & $\begin{array}{c}\text { Secondary } \\
\text { impact in } \\
\text { grid cell } 1 / \\
\text { total } \\
\text { emission }\end{array}$ & $\begin{array}{l}\text { Secondary } \\
\text { impact in } \\
\text { grid cell } 2 / \\
\text { total } \\
\text { emission }\end{array}$ & $\begin{array}{l}\text { Secondary } \\
\text { impact in } \\
\text { grid cell 3/ } \\
\text { total } \\
\text { emission }\end{array}$ & $\begin{array}{l}\text { Secondary } \\
\text { impact in } \\
\text { grid cell } 4 / \\
\text { total } \\
\text { emission }\end{array}$ & $\begin{array}{c}\text { Secondary } \\
\text { impact in } \\
\text { grid cell 5/ } \\
\text { total } \\
\text { emission }\end{array}$ \\
\hline Case 1 & $4.7 \%$ & $42.9 \%$ & $57.1 \%$ & 0 & 0 & 0 \\
\hline Case 2 & $24.8 \%$ & $2.8 \%$ & $65.3 \%$ & $31.9 \%$ & 0 & 0 \\
\hline Case 3 & $19.6 \%$ & $3.8 \%$ & $76.9 \%$ & $19.3 \%$ & 0 & 0 \\
\hline Case 4 & $57.4 \%$ & $0.6 \%$ & $28.6 \%$ & $55.1 \%$ & $13.3 \%$ & $2.4 \%$ \\
\hline
\end{tabular}




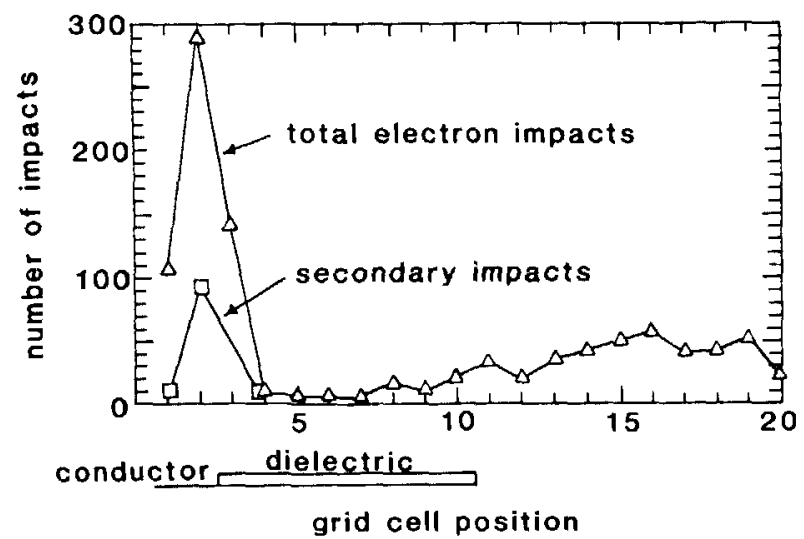

FIG. 4. For case $2(70 \mathrm{~V}$, s yield of 4.2), total electron impacts and secondary impacts vs grid cell position. The impact data for the entire grid cell shown at grid cell center.

ever, this may be a function of the computer code and not a physical result.

\section{COMPARHSONS AND DISCUSSHON}

\section{Case 1}

The first case is not very interesting in terms of secondary emission. Snapover has not been reached so the Kapton surface has a negative potential. Very few electrons strike the dielectric surface, only 9 on the first dielectric cell compared to 61 and 88 on the two conductor cells, respectively. The nine electrons appear even more insignificant when the area effect is taken into account. The grid cells actually represent concentric rings; thus the area of the first is approximately one-third the area of the second and one-fifth the area of the third. Electrons that strike the dielectric have energy that decreases with distance from the conductor, due to the decreasing potential through which they are accelerated. In this case only seven secondaries are emitted-all from the first dielectric grid cell. The electrons that strike further out on the dielectric do not have enough energy to emit secondaries. This is indeed a small effect; $4.7 \%$ of the current is accounted for by secondaries (see Table I). Taking the area effect into account, two-thirds of the electrons strike the first cell. As will presently be shown, this peak moves toward the second conductor cell and then to the first dielectric cell as both voltage and s yield are increased.

\section{Case 2}

In case 2 secondary emission is significant. Snapover has just been reached, which is evident from the positive potential on the first cell of the dielectric surface (see Fig. 3, upper right). The total current to the conductor is larger than in the previous case by a factor of 2.7. This is explained by the increased voltage and the secondary emission effect. Without secondaries the increase in current would only be a factor of 2.0 arising from the increased voltage. The remainder would be accounted for by secondary emission. As can be seen from Fig. 4 or Table I, 25\% of the current is due to secondaries, and almost all of the secondaries impact on the second conductor cell. Thus, the secondaries contribute to the outward shift of the peak electron impact mentioned earlier.

In case 1, taking the area effect into account, approximately $66 \%$ of the current strikes the first grid cell. In case 2 , without secondaries, the result is similar $(61 \%)$. With secondaries, only $52 \%$ of the current impacts on the first conductor cell-about half of the current is accounted for by each conductor cell. The increase in the second cell is due to secondaries. The explanation for this particular increase lies in the electric field strength. The secondaries move in the electric fields in the same way as incident electrons. Since secondaries usually have low kinetic energies, they are pulled toward the conductor or back to the dielectric very quickly. This results in the hopping motion along the dielectric mentioned earlier.

\section{Case 3}

Case 3 is at a higher s yield but lower voltage than case 2. The percentages are similar, but the total number of impacts and emissions is about half for case 3. This indicates that voltage increase is more significant than is the s yield increase. The secondary emission model is distinctly energy dependent as seen from Eq. (1). The maximum yield occurs at $155 \mathrm{eV}$ so it would be expected in a purely energy-dependent model that a 70-eV electron would emit more secondaries than a 40-eV electron. The secondary emission model, however, is also dependent on $\mathrm{s}$ yield so that a $70-\mathrm{eV}$ electron at $s$ yield of 4.2 actually emits fewer secondaries than a $40-\mathrm{eV}$ electron at $s$ yield of 8.4. A further explanation might lie in the importance of a sheath region to the collection of current. Brandon ${ }^{2}$ found that the current collected by the exposed conductor is proportional to the area of the sheath and that the area of the sheath is directly related to the potential on the conductor. Comparing cases 2 and 3 , we see twice as much current collected in the former. Thus we expect the sheath region to be twice as large. In case 3 not as many electrons "see" the conductor; instead their motions are dominated by thermal energy. The electrons are shielded so fewer can get to the conductor or to the dielectric to emit secondaries that will add to the current. For this reason voltage is the dominant factor.

The current enhancement due to secondaries is $20 \%$. This can be compared to case 2 which is also at the onset of

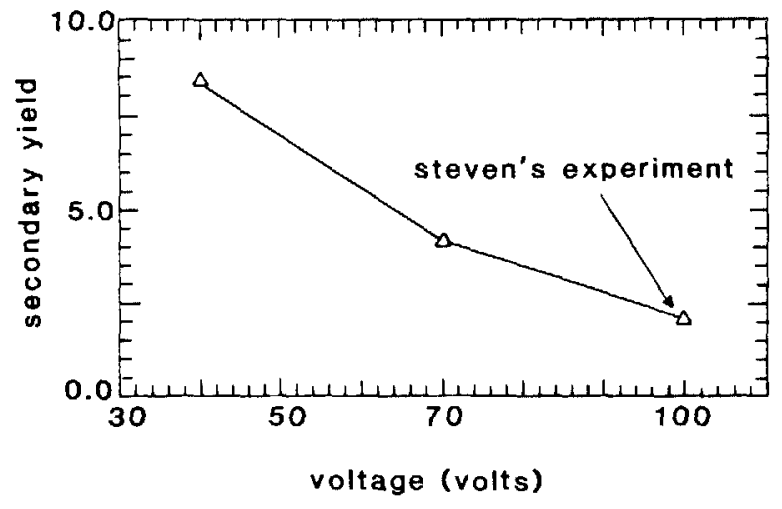

FIG. 5. Secondary yield vs voltage at the onset of snapover. 


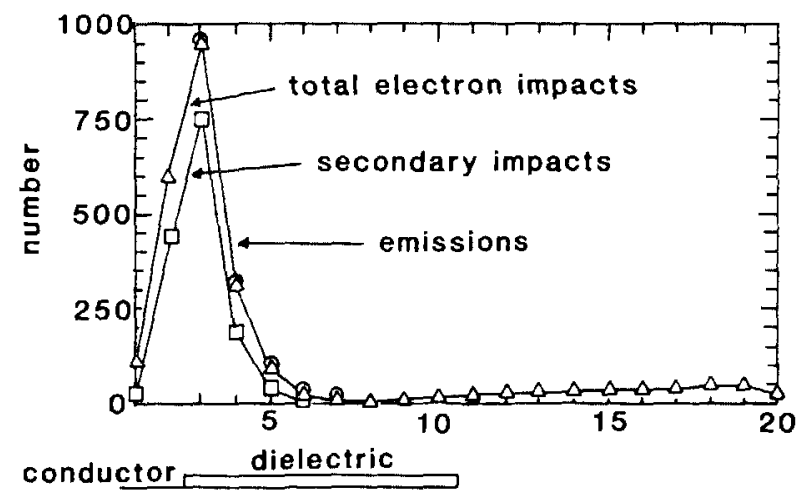

grid cell position

FIG. 6. For case 4 ( $70 \mathrm{~V}$, s yield of 8.4), impacts and emissions vs grid cell position. The data for the entire grid cell shown at grid cell center.

snapover and has a current enhancement of $25 \%$. Table $I$ is a listing of various percentages. Notice that cases 2 and 3 are quite similar; few secondaries impact on the first conductor cell; the majority impact on the second conductor cell. Figure 5 is a plot of voltage versus s yield. Cases 2 and 3 are represented by points on this graph. The third point at $100 \mathrm{~V}$ is from an earlier study (not reported here) and agrees with the Stevens experiment discussed earlier. The points are connected by lines to give an approximation of the curve for the onset of snapover. Along this curve, the points will have similar characteristics such as the percent increase due to secondaries and the position of impact; however, the total current will increase as the voltage increases. Values of $\mathrm{s}$ yield above 8.4 are not realistic. In fact 8.4 is stretching the limit, but since this point was discussed by Brandon, ${ }^{2}$ it is also presented here. For $100 \mathrm{~V}$ and above, a larger number of grid elements must be used in the simulation which is also a limiting factor. These four cases were run on a CRAY-I computer which can accommodate a $60 \times 60$ run at 18000 particles. Future runs will all be made on a VAX 11-750 which can easily handle $10 \times 10$ runs and can less easily handle $20 \times 20$ runs, but cannot handle $40 \times 40$ runs.

\section{Case 4}

As expected of a higher s yield and voltage, the current is greatly enhanced for case 4. This case is well into snapover. More of the dielectric surface is at a positive potential as can be seen from Fig. 3, lower right. This causes more electrons to impact on the dielectric surface with sufficient energy to emit secondaries. In this case, $57 \%$ of the current is due to secondaries. With a further increase in potential the conductor hole could be essentially shielded from incident electrons, and the current would be due solely to secondaries. Figure 6 shows the impacts and emissions as functions of distance along the $R$ axis. In this case the total electron impacts are greatest on the first dielectric cell. When the area effect is taken into consideration, the second conductor cell has slightly more impacts than the first dielectric cell, but the first conductor cell has half the value. The impact peak has shifted as was suggested earlier. This is due to the hopping motion along the dielectric. This can be seen more clearly from Fig. 7, which is a finer scale of the same impacts and emissions but only along the dielectric surface. The curves for total electron impacts and electron emission are similar, but the secondary impacts, which must account for all the emissions, assuming no secondary escapes, are shifted toward the conducting hole. The maximum of all three curves is at the conductor-dielectric edge. Total electron impacts are at first greater, then less, and then greater than electron emissions. The fact that the electrons are actually macroelectrons and have size associated with them helps to explain this. When electrons impact on the first dielectric cell, the charge is spread over both the conductor and the dielectric so fewer secondaries are emitted. However, the incident electrons have sufficient energy to emit more than one secondary as can be seen from the emission curve rising above the impact curve between grid cells 3 and 4, where less incident charge is shared with the conductor. In moving further out on the dielectric surface, the incident energy is decreased and fewer secondaries are emitted per incident electron. The secondary impact curve is shifted toward the conductor hole which shows that electrons emitted along the dielectric surface only travel a few grid cells at a time, on the average, before impacting on the surface. This illustrates the motion of hopping along the dielectric surface which was discussed earlier.

\section{SUMMARY AND CONCLUSIONS}

Stevens suggested that at a certain positive voltage enough secondaries were emitted to enhance the current. Brandon attributed this to the higher voltage spreading onto the dielectric surface and causing incident electrons to be pulled to the dielectric with sufficient energy to emit secondaries. This study supports these ideas with enhanced diagnostics to pin down the sources and sinks of electrons and to explain the mechanisms in greater detail. The following points can be made:

(1) The impact maximum moves outward from the conductor hole with increasing values of voltage and $s$ yield: in case 1 the peak is on the first conductor cell; in cases 2 and 3 the peak is between the first and second conductor cells; and in case 4 the peak is on the second conductor cell. It is suggested that with a further voltage increase the peak would move onto the dielectric surface.

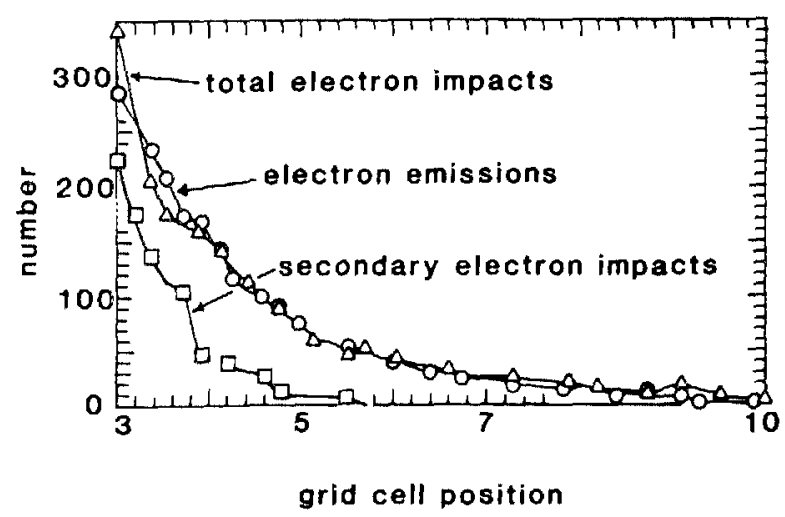

FIG. 7. For case 4, finer-scale view over the dielectric surface showing impacts and emissions vs grid cell position. 
(2) As the peak moves outward toward the dielectric, more of the current is due to secondaries: $5 \%$ for case $1 ; 25 \%$ and $20 \%$ for cases 2 and 3, respectively; and $57 \%$ for case 4 .

(3) Runs at the onset of snapover exhibit similar characteristics such as the percent increase due to secondaries and the position of impact, but the total numbers of impact and emission increase with increasing voltage along the curve.

(4) Voltage increase is the dominant mechanism for current increase. The voltage spreads out further into the simulation space causing more particles to "see" the conductor. At lower voltages a large number of particles are outside the shielded region and either do not impact on the dielectric or impact on the outer edge without sufficient energy to emit secondaries.

(5) Secondaries that are emitted have sufficient energy to travel only a few grid cells before being pulled to the conductor or back to the dielectric, which results in a motion of hopping along the dielectric surface.

\section{ACKNOWLEDGMENTS}

The authors wish to thank Dr. S. T. Brandon for guidance in a previous collaboration that eventually led to this study, Dr. J. Enoch for his helpful suggestions, and M. Allen for important technical assistance. Invaluable run time on a CRAY-I computer was provided by United Telecom's Computer Service Group, Kansas City, Missouri. This work was supported in part by NASA Lewis Research Center, Research Grant NSG-3290.

'N. J. Stevens, Spacecraft Charging Technology Conference (NASA CP2071, Washington DC, 1978).

'S. T. Brandon, Ph. D. thesis, University of Kansas, 1984 (unpublished).

${ }^{3}$ S. T. Brandon, R. L. Kessel, J. Enoch, and T. P. Armstrong, J. Appl. Phys. 56, 3215 (1984).

${ }^{4}$ J. H. Nonnast and J. Enoch, J. Appl. Phys. 54, 621 (1983).

${ }^{5}$ R. C. Chaky, J. H. Nonnast, and J. Enoch, J. Appl. Phys. 52, 7092 (1981).

J. H. Nonnast, R. C. Chaky, T. P. Armstrong, J. Enoch, and G. C. Wiseman, Spacecraft Charging Technology-1980 (NASA CP-2182, Washington, DC, 1981)

'R. C. Chaky, J. H. Nonnast, T. P. Armstrong, J. Enoch, and G. C. Wiseman, Spacecraft Charging Technology-1980 (NASA CP-2182, Washington DC, 1981).

${ }^{8}$ J. W. Haffner, Spacecraft Charging Technology Conference (NASA CP. 2071, Washington, DC, 1978). 